Mal J Nutr 25(3): 377-391, 2019

\title{
Insights into knowledge, attitude and practices on early complementary feeding of infants among Saudi mothers
}

\author{
Atheer Aldossari, Dalal Alshehri, Ghadah Alzahrani, Salma Almasoud, Yousr \\ Alaqeel \& Nada Benajiba*
}

Clinical Nutrition Programme, Department of Health Sciences, College of Health and Rehabilitation Sciences, Princess Nourah Bint Abdulrahman University, Saudi Arabia

\begin{abstract}
Background: The World Health Organization recommends that mothers avoid early complementary feeding of infants before the age of 6 months, to promote maximum growth and health. However, this practice is still high among Saudi mothers. This study aimed to assess knowledge, attitude and practices (KAP) towards early complementary feeding among Saudi mothers in Riyadh. Methods: A crosssectional study was conducted in Riyadh city among 771 mothers of children, aged 6-24 months. Data was collected through an online questionnaire that assessed KAP aspect regarding early complementary feeding among mothers. Results: More than one-third of mothers (37.4\%) had introduced early complementary feeding, out of which $83.3 \%$ later stated that the main reason for this was because they thought that the baby was old enough to receive complementary foods. The two most common types of complementary foods given to the baby before 6 months were liquids $(83.0 \%)$ and mashed foods $(72.9 \%)$. The mothers who introduced complementary feeding before 6 months of age compared to those who initiated complementary feeding after 6 months were observed to have a higher percentage of medium knowledge and neutral attitude $(76.0 \%$ versus $54.4 \%$ and $55.2 \%$ versus $62.5 \%$, $(p<0.05)$, respectively). Mothers' knowledge and attitude significantly influenced their practices in relation to early complementary feeding $(p<0.05)$. Conclusion: The findings demonstrated that the appropriate knowledge and attitude are important to promote the introduction of complementary feeding at 6 months in the population that was studied. Thus, education on complementary feeding should be promoted.
\end{abstract}

Keywords: Knowledge-attitude-practices, complementary feeding before 6 months, Saudi mothers

\section{INTRODUCTION}

Nutrient adequacy is crucial in the periods of infancy and early childhood, as it is necessary for ideal growth, health and development. The World Health Organization (WHO, 2003), has stated that complementary feeding should start when breast milk alone is no longer sufficient to meet the nutritional requirements of infants, and therefore other food and liquids are needed, along with breast milk. It has recommended that mothers breastfeed their infants from birth until they reach the age of 2 years and that complementary feeding should not be introduced before 6 months of

\footnotetext{
*Corresponding author: Dr. Nada BENAJIBA

College of Health and Rehabilitation Sciences, Princess Nourah Bint Abdulrahman University

P.O. Box 84428, Riyadh 11671, Kingdom of Saudi Arabia

Tel: 00966118240752; Mobile: 00966537504950; Email: benajibanada@gmail.com

doi: https://doi.org/10.31246/mjn-2019-0072
} 
age. Thus, early complementary feeding is the introduction of foods other than breastmilk to infants before the age of 6 months (WHO, 2003).

Infants who had been exposed to early complementary foods (before 6 months) were at higher risk of consuming foods and fluids that had microbial contamination (Tang, Lee \& Binns, 2015). According to a study conducted in India, infants under 6 months of age were showed to have gastrointestinal system, kidneys and neurodevelopment that were not fully developed (Ip et al., 2009). Another study concluded that infants who were fed exclusively with formula had a higher risk of developing acute otitis media compared to those infants who were exclusively breastfed (Rao et al., 2011). In several countries of Middle East and North Africa region (the so-called MENA region), such as United Arab Emirates (UAE), Lebanon, Kuwait, Libya, Egypt, Bahrain and Yemen some mothers introduce non-milk fluids such as sweetened water and herbal teas to their infants who are under 6 months. Such a practice also appeared to be related to diarrhoea in infancy (Radwan, 2013; Batal, Boulghourjian \& Akik, 2011; Al-Awadi \& Amine, 1997; Shembesh, Balo \& Singh, 1997; El Mougi et al., 1981; Al-Sairafi \& Al-Dallal, 2002; Central Statistical Organization, 1994). In addition, a systematic review revealed that infants who had been exposed early to solid foods were at higher risk of being overweight in childhood (Pearce, Taylor \& Langley-Evans, 2013). The knowledge of appropriate nutrition of mothers has been demonstrated to have an important role on how the child will be fed. However, a study done in Kosova showed that although $88.4 \%$ of mothers had sufficient knowledge of complementary feeding, only $38.4 \%$ of them were willing to apply that knowledge (Berisha et al., 2017).
In terms of practice, research studies on complementary feeding conducted in the Kingdom of Saudi Arabia (KSA) and Kuwait showed that the recent feeding practices were falling behind in their compliance with the WHO recommendations (El Mouzan et al., 2009; Scott et al., 2015). An earlier study done in the city of Riyadh in the KSA found that $51.4 \%$ of infants were formula fed by 1 month of age and 90\% by 6 months of age, while $80.8 \%$ were introduced to solid food at the age of 4-6 months (El Mouzan et al., 2009).

The theory of planned behaviour describes the linkage between knowledge, attitude and practices (KAP) among people in health studies (Manderson \& Aaby, 1992; Launiala, 2009). There are a few studies on early complementary feeding in Saudi Arabia but none on early complementary feeding that have explored KAP of mothers in Saudi Arabia. This study was conducted to investigate the KAP of early complementary feeding among mothers in Riyadh.

\section{MATERIALS AND METHODS}

\section{Study design and subjects}

This cross-sectional study was undertaken in Riyadh city, from January to March 2019. A total of 771 Saudi mothers participated in the study. They fulfilled the following inclusion criteria: resident in Riyadh, were at procreative age (18-45 years old), have had a child age 6-24 months, have had infants without chronic diseases or metabolic disorders or health complications and were mothers of full-term infants. Ethical approval was obtained from the Institutional Review Board of Princess Nourah Bint Abdulrahman University (H-01-R-059/ 18-0364). All participants were fully aware of the requirements for participation and were informed that their participation was voluntary. 
Data was collected using a convenient sampling technique through an online questionnaire.

\section{Research tools}

The KAP questionnaire was adapted from a previously validated questionnaire (Kittisakmontri et al., 2018) and translated to Arabic and then translated back to English. It was modified before the back translation to meet the objectives of this study. To guarantee clear and ease of understanding by the participants, the questionnaire was tested among 23 individuals. Section 1 of the questionnaire contained questions about general information of mothers and demographic characteristics of the infants, such as mother's age, number of children level of education, occupation, income, child's gender, and age. Section 2 of the questionnaire focused on measuring KAP, as follows:

- Knowledge: questions on the knowledge of the mothers on the appropriate age for the introduction of complementary foods, the types of foods introduced prior to 6 months and their source(s) of information.

- Attitude: questions that assessed the attitude of mothers towards the introduction of liquids and semisolid foods, and the sufficiency of breast milk to their infants before the age of 6 months.

- Practices: this assessment contained questions on the age of the infant at which mothers started the introduction of complementary foods, reasons that drove them to initiate early complementary feeding, the texture and characteristics of the early foods that were introduced.

To assess the interrelationship between the three aspects of KAP, a score was assigned for each of them.

Both knowledge and attitude were divided into three equal levels by dividing the total score of each aspect on $33.3 \%$ for the lowest level, 33.4-66.6\% for medium level and $66.7-100.0 \%$ for the highest level. Categorisation was based on the total score obtained for each aspect. For knowledge, each correct and wrong answer was scored as 1 point and 0 point, respectively. Answers for attitude ranged from strongly disagree to strongly agree. These were scored as 4 points for strongly disagree, 3 point for disagree, 2 points for neutral, 1 point for agree and zero point for strongly agree. For practices, categorisation was based on the answer to the question, "when did you introduce complementary food to your child?". Score was 0 and 1 for introducing the complementary feeding before and after 6 months of age of the infant, respectively.

\section{Statistical analysis}

The data was collected from the questionnaire, entered and analysed using the SPSS programme version 21. The total population was divided into two groups, based on the time complementary feeding was initiated: Group $1 \quad$ (<6 months) defined as mothers who started introducing complementary feeding before 6 months of infant's age $(n=288)$; and Group 2 (>6 months), defined as mothers who started introducing complementary feeding after 6 months of infant's age $(n=483)$. Descriptive statistics were presented as percentage (\%). To assess the differences between the two groups and the interrelationship between KAP variables, the Pearson correlation and chi-square tests were used. The results were considered statistically significant when $p$-value $<0.05$.

\section{RESULTS}

\section{Social demographic}

Of a total of 771 mothers who participated in the study, 37.4\% (288) 
had introduced early complementary feeding while $62.6 \%$ (483) had introduced complementary feeding after 6 months of the infant's age. In terms of the sociodemographic data, results showed that majority of mothers who introduced early complementary feeding were those with diploma or bachelors degree educational level $(71.9 \%)$ and mothers with firstborn infant $(28.5 \%)$. However, there was no significance in the socio-demographic data between mothers who introduced complementary feeding before and after 6 months of age (Table 1).

\section{Knowledge}

Our results in Table 2 showed that the majority of the participants $(65.5 \%)$ had chosen 6 months or later as an appropriate age for the introduction of complementary feeding among all mothers, indicating that they possessed knowledge that matched the WHO recommendation. In addition, $73.9 \%$ agreed that the most important factor to promote infant growth was exclusive breastfeeding for at least 6 months. Among the total of $483(62.6 \%$ of all mothers) who introduced complementary feeding after 6 months or later, $90.9 \%$ of them chose 6 months as the appropriate age to start. Of a total of 288 mothers (37.4\% of all mothers) who had introduced early complementary feeding, $40.9 \%$ of them had chosen 4 months as an appropriate age to start introducing complementary food.

There was a significant difference in the knowledge of mothers about the time for the introduction of semi-solid foods. The proportion of mothers who chose semi-solid as an appropriate food to be added before 6 months of age was $63.2 \%$, whereas $34.0 \%$ chose semi-solid food as an inappropriate food to be added before 6 months of age $(p=0.000)$.

There was no significant difference in knowledge in the introduction of liquid $(p=0.290)$ and solid foods $(p=0.175)$ between the two groups. On the introduction of liquid and semisolid food during the first six months of infant's age, differences were observed based on the different types of complementary food: for example, juice, yoghurt, cereals and mashed foods were $55.6 \%, 56.9 \%, 81.9 \%$ and $74.0 \%$, respectively. Most answers of mothers indicated that sugar and salt should not be added to the infant's complementary food before the age of 6 months based on their knowledge by $83.7 \%$ and $90 \%$ respectively. Notably, $54.2 \%$ of the first group ( $<6$ months) and $58.4 \%$ of the second group (>6 months) were aware that healthcare professionals were the most reliable source of information about early complementary feeding (Table 2).

\section{Attitude}

Among the first group ( $<6$ months), $57.2 \%$ (the total percentage of agree and strongly agree) had a positive attitude towards starting early complementary feeding, while in the second group (>6 months), $67 \%$ (the total percentage of disagree and strongly disagree) had a negative attitude towards starting early complementary feeding. Of the first group ( $<6$ months) $26.7 \%$ indicated that breast milk alone was not enough to meet the infant's nutritional requirements and $40.0 \%$ felt that it did not meet the water requirement as well. In contrast, $74.3 \%$ of the second group (>6 months) agreed that it (breast milk) was enough to cover the infant's nutritional requirement and $59.6 \%$ of them believed that water should not be introduced before 6 months of age. Similarly, on mothers' knowledge, both groups agreed that sugar and salt should not be added to an infant's complementary food before 6 months by $80.6 \%$ and $81.5 \%$, respectively (Table 3). 
Table 1. Socio-demographic characteristics of mothers and infants based on the time of introducing complementary feeding before and after 6 months

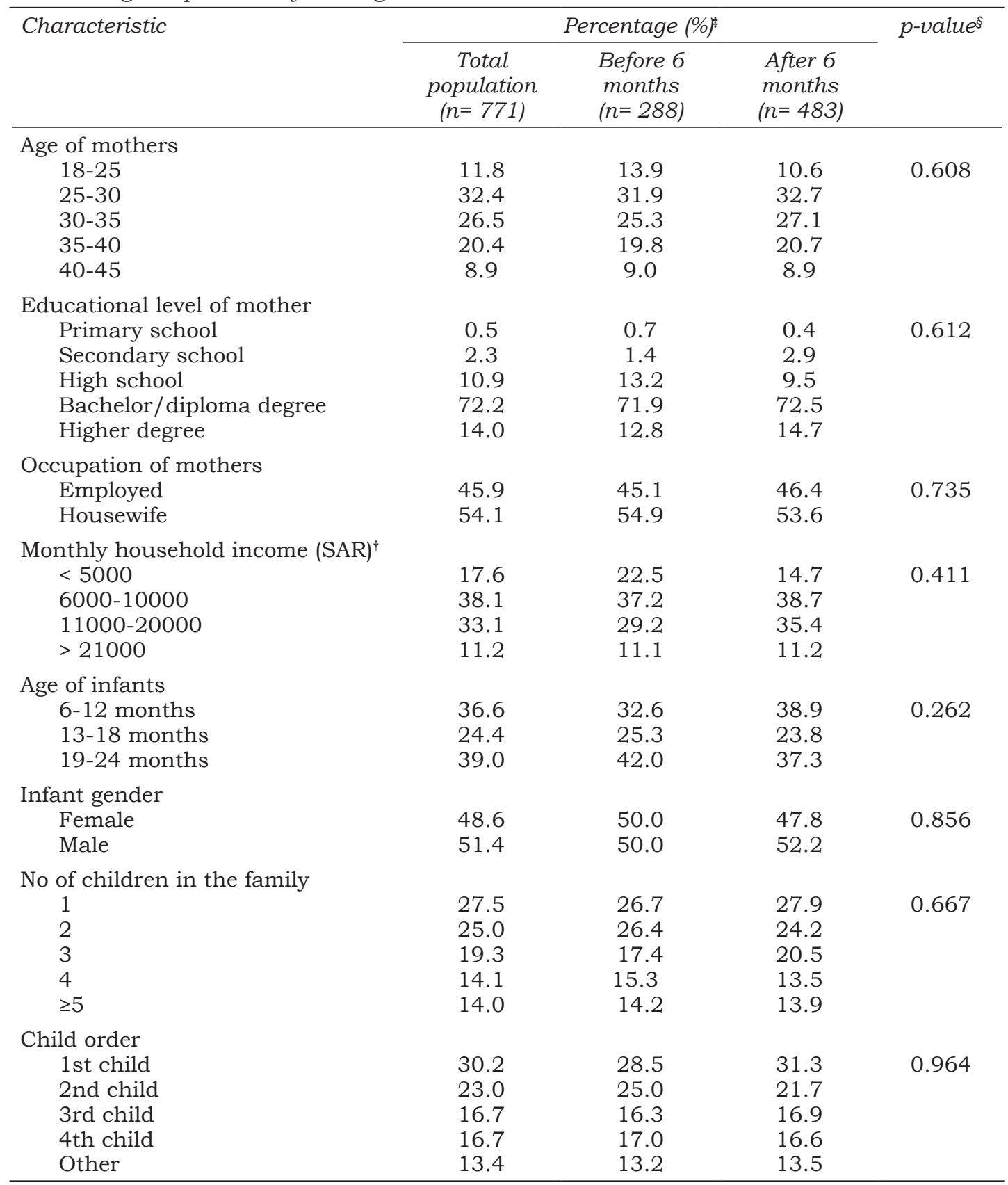

${ }^{\dagger}$ SAR = Saudi Riyals; 1 Saudi Riyal $=0.26$ \$US

¥Percentages were calculated based on total population of each group $s_{\mathrm{p}}$-value was calculated by chi-square test 


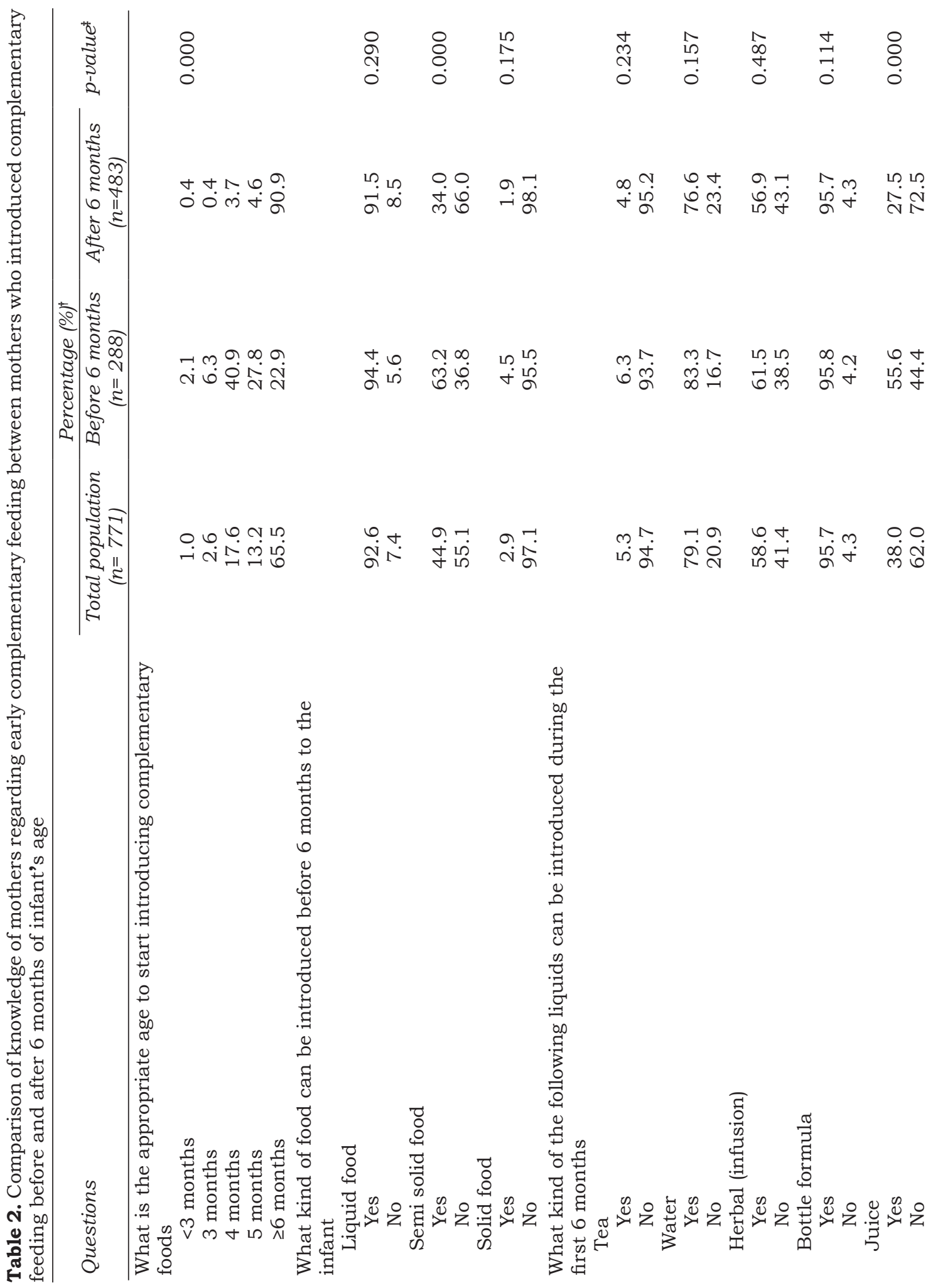


$\begin{array}{llllllll}0 & 0 & 0 & 0 & 0 & 0 \\ 0 & 0 & 0 & 0 & 0 & 0 & 0 & 0 \\ 0 & 0 & 0 & 0 & 0 \\ 0 & 0 & 0 & 0 & 0\end{array}$

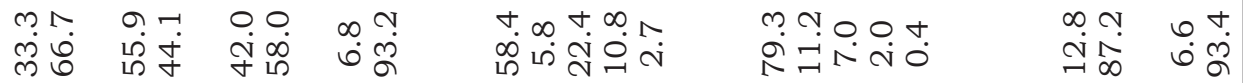

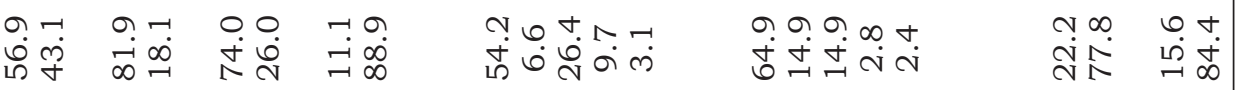

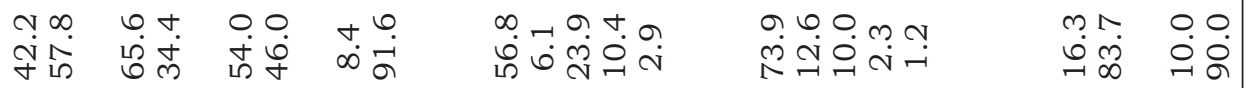

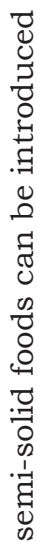

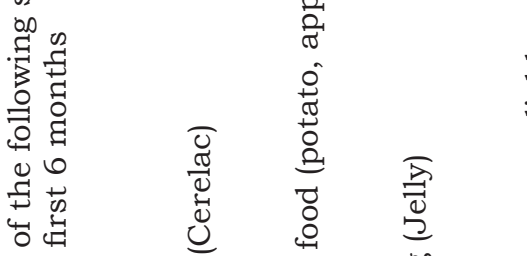

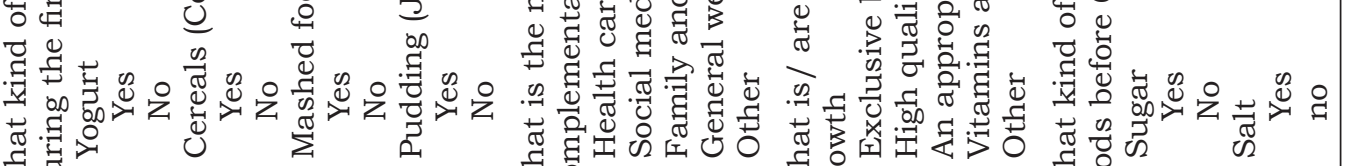
光正 0

今

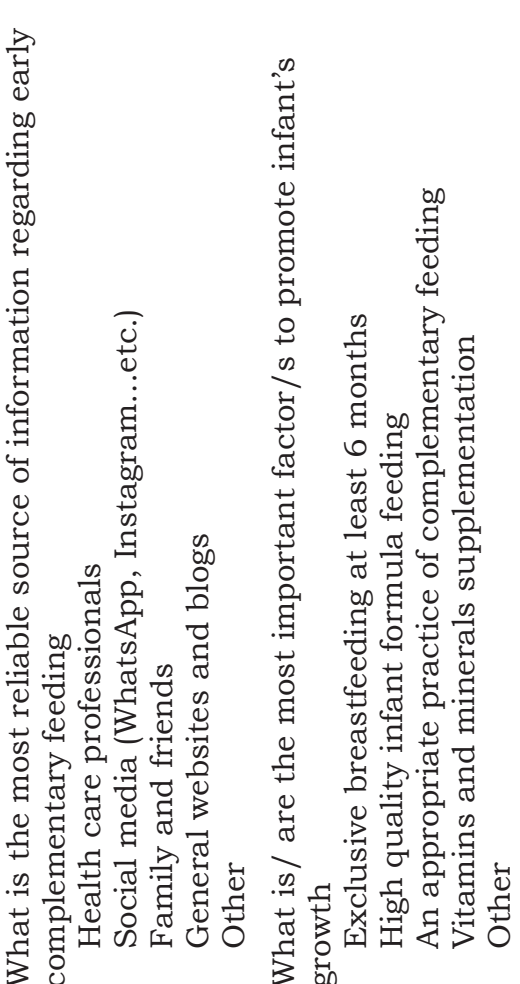

ت

节

동

(4)

胥

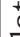

วั

站

융

도 己

D.

\%

동

营

글

ฮై

넌 ᄃㅇㅇㅇ 


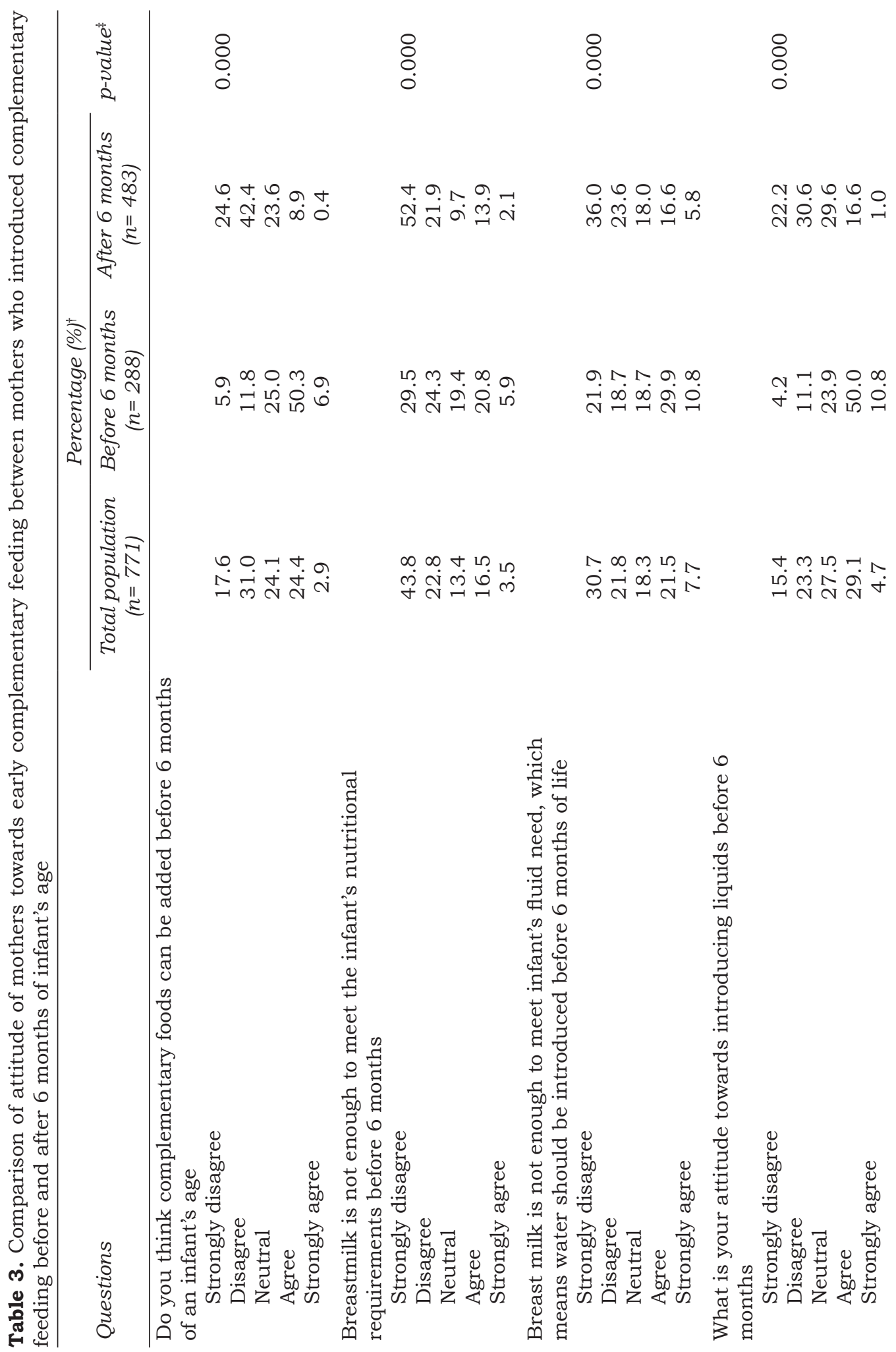


$ᄋ$
0

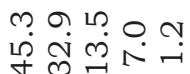

ㄱ. m.

ণั

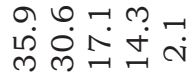

3
0
0

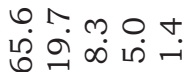

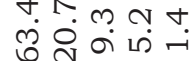

ก ก .

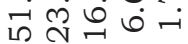

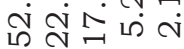

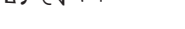

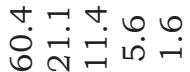

ㄴ. ㄴำ ㄴำ
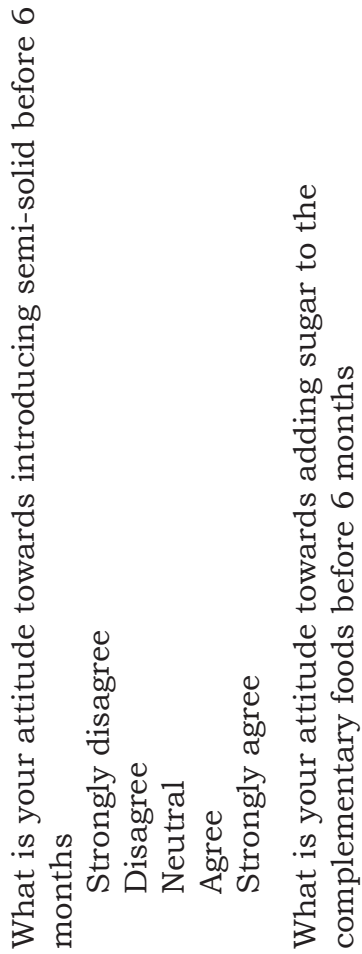

운

$\infty_{\infty}^{\infty}$

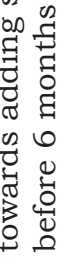

\% 50

盛

क्षे

క్

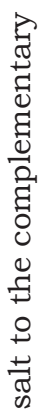

䓀

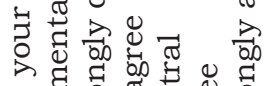

\& ${ }_{0}$

ชั

3

क व 0 \%

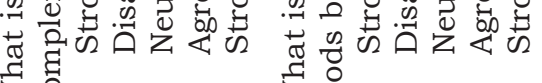
今

3 


\section{Practices}

In regard to practices (Table 4), the results revealed that $37.4 \%$ of mothers introduced complementary food to their infants before the age of 6 months. Among mothers of the first group ( $<6$ months), the majority introduced complementary food to their infants in the fourth or fifth month of age $147.5 \%$ and $45.5 \%$, respectively). Of the reasons given, $83.3 \%$ of them stated that the main factor that drove them to initiate early complementary feeding was that they felt the baby was old enough to receive complementary foods. Among all the mothers surveyed, $55.9 \%$ gave a combination of breast milk and infants formula during the first 6 months of age. Of the most common two types of food given to the baby before 6 months, $83.0 \%$ favoured liquids and $72.9 \%$ preferred mashed foods. Vegetables and fruits, carbohydrate foods, and dairy products were the most likely food groups given to the infant before they reached 6 months of age by $85.8 \%, 61.1 \%$ and $54.9 \%$ respectively. On the other hand, sugar was more likely to be added than salt. The results showed $20.1 \%$ of mothers added sugar while $17.4 \%$ added salt (Table 4).

\section{Scoring and interrelationship between knowledge, attitude and practice}

As summarised in Table $5,62.5 \%$ of the total study population appeared to possess a medium level of knowledge. The majority of both groups, viz. $<6$ months and $>6$ months, had medium knowledge with percentages of $76.0 \%$ and $54.4 \%$, respectively. However, there was an obvious difference for those with high knowledge with only $10.0 \%$ for the first group of mothers $(<6$ months) showing this feature and $43.7 \%$ for the second group (>6 months).

As for attitude, the results indicated that the majority of all the mothers $(55.2 \%)$ had a higher tendency towards neutral attitude towards the early introduction of complementary feeding. However, $62.5 \%$ of the mothers in the first group ( $<6$ months) showed a neutral attitude towards initiating early complementary feeding. It was observed that $68.3 \%$ of the second group $(>6$ months) believed that complementary feeding should be started at the age of 6 months or later. This meant that they had a negative attitude towards early complementary feeding.

Overall, scores for practices showed that $62.6 \%$ of the total population surveyed were good while $37.4 \%$ had poor practices. Regarding the association between the 3 aspects (KAP) among the total population surveyed, results showed that knowledge and attitude had significantly correlated to practice $(p$-value $=0.000)$ (Table 5) .

\section{DISCUSSION}

This KAP study aimed to examine the current state of early complementary feeding among Saudi mothers in Riyadh. The most important result was that $37.4 \%$ of the 771 mothers who were studied had introduced early complementary feeding to their infants. This figure was lower compared reported in a study done in Tabuk which showed that $62.5 \%$ of mothers introduced complementary feeding before the child reached 4 months of age (Alzaheb, 2016). The reasons for these findings may be due to their children getting hungry, the perception that it was a good age to start complementary feeding besides other factors. Another study conducted in Saudi Arabia that included five different regions (Northern, Southern, Middle, Eastern and Western), reported that $83 \%$ of infants were introduced to early complementary feeding (Adam \& Osama, 2019).

In this study, higher percentages of medium knowledge, neutral attitude 
Table 4. Practice of mothers regarding early complementary feeding among mothers who introduced complementary feeding before 6 months of infant's age $(n=288)$

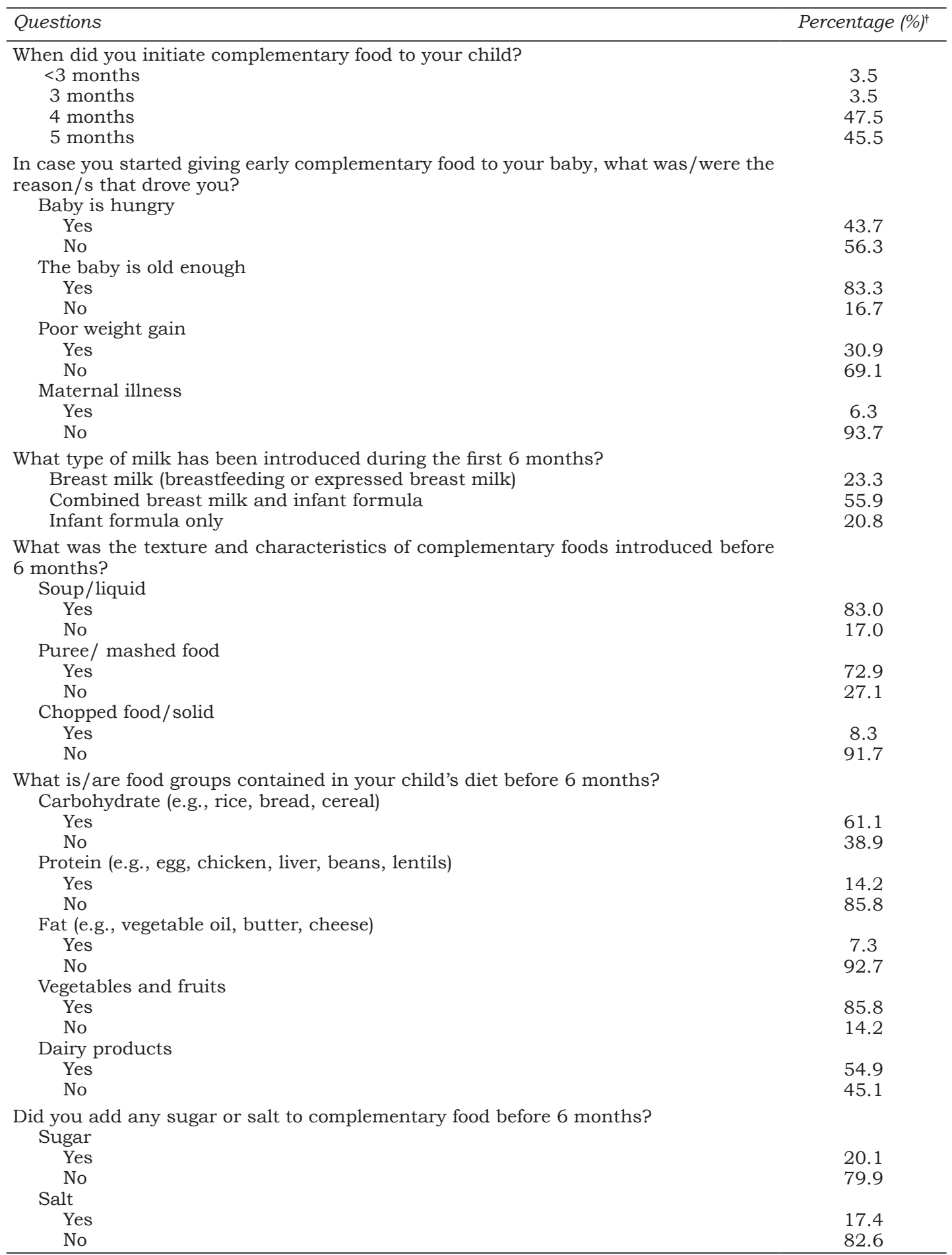

${ }^{\dagger}$ Percentages were calculated based on population of mothers who introduced early complementary feeding $(n=288)$ 
Table 5. Comparison of scoring of knowledge, attitude and practice of mothers towards early complementary feeding between mothers who introduced complementary feeding before and after 6 months of infant's age

\begin{tabular}{|c|c|c|c|c|}
\hline \multirow[b]{2}{*}{ Aspects } & \multicolumn{3}{|c|}{ Percentage $(\%)^{\dagger}$} & \multirow[b]{2}{*}{$p$-value } \\
\hline & $\begin{array}{l}\text { Total population } \\
\qquad(n=771)\end{array}$ & $\begin{array}{c}\text { Before } 6 \text { months } \\
(n=288)\end{array}$ & $\begin{array}{l}\text { After } 6 \text { months } \\
\quad(n=483)\end{array}$ & \\
\hline \multicolumn{5}{|l|}{ Knowledge } \\
\hline Low & 6.3 & 14.0 & 2.0 & Versus Attitude $=0.000$ \\
\hline Medium & 62.5 & 76.0 & 54.4 & Versus Practice $=0.000$ \\
\hline High & 31.0 & 10.0 & 43.7 & \\
\hline \multicolumn{5}{|l|}{ Attitude } \\
\hline Negative & 13.6 & 14.5 & 7.25 & Versus Knowledge $=0.000$ \\
\hline Neutral & 55.2 & 62.5 & 24.4 & Versus Practice $=0.000$ \\
\hline Positive & 31.0 & 23.0 & 68.3 & \\
\hline \multicolumn{5}{|l|}{ Practice } \\
\hline Poor & 37.4 & 100.0 & 0.0 & Versus Knowledge $=0.000$ \\
\hline Good & 62.6 & 0.0 & 100.0 & Versus Attitude $=0.000$ \\
\hline
\end{tabular}

${ }^{\dagger}$ Percentages were calculated based on total population of each group

${ }^{\ddagger} p$-value testing the interrelationship between the knowledge, attitude and practice calculated by chi-square test

and poor practices regarding early complementary feeding were observed among the group of mothers who introduced early complementary feeding to their infants compared to the mothers who had initiated complementary feeding after 6 month of age. In addition, knowledge and attitude of the mothers significantly influenced their practices in relation to early complementary feeding.

Azzeh (2017) found that the practice of complementary feeding was significantly associated with the job patterns of the mothers as well as the educational level of the parents. In contrast, there was no significant association between education and the age of mothers with the time of the introduction of complementary feeding in Kosova (Berisha et al., 2017). The finding from the Kosova study matches the result of this study, which demonstrated no significant difference between starting early or on time complementary feeding in terms of socio-demographic characteristics of mothers in both groups. This might be related to the differences in the level of knowledge. The appropriate age for the introduction of complementary feeding was known among $65.5 \%$ of the current study. Similarly, studies from Ethiopia, Abha, and India revealed that $60.3 \%$, $89.3 \%$ and $92.5 \%$ respectively had the right knowledge about starting timely complementary feeding (Reda, Teferra \& Gebregziabher, 2019; Khattab, 2000; Jain, Thapar \& Gupta, 2018). As with Ethiopia, the educational level of the husbands allowed them to support their wives to delay early weaning for better health of the child. In Abha and India, the higher educational level of mothers positively influenced their knowledge.

As for knowledge, $56.8 \%$ stated that the most reliable source of information about complementary feeding were health care professionals. A study conducted in Erbil city showed mothers learnt about infant feeding from physicians (41.6\%) and from nurses (54.7\%), at the primary healthcare centers (Al-Azzawi, Hussein \& Shaker, 2012). A study conducted in Saudi Arabia showed that 
mothers learnt about breastfeeding from medical personnel $(44.9 \%)$ and from their relatives $(26.8 \%)$ (Al-Jassir et al., 2006). Thus, the role of health care as knowledge providers should be seriously promoted in order to ensure that correct and adequate information on breastfeeding practices is provided to mothers.

In regard to attitude and practices, $83.3 \%$ of mothers in the current study and $26.6 \%$ in a previous study conducted in Tabouk showed that complementary feeding was initiated when mothers thought that the baby was old enough to receive complementary foods (Alzaheb, 2016). A study in Abha found that $63.1 \%$ of mothers believed that water and fluids were needed for the infant during the first 4 months of age (Khattab, 2000). However, only $29.2 \%$ of the current study population believed that water and fluids were needed in addition to breast milk before the age of 6 months. The majority of mothers $(66.6 \%)$ in a study conducted in Nigeria added salt to the meals of their infants (Olatona et al., 2017), whereas only $17.4 \%$ of mothers did so before 6 months in our study. This disparity in practices may relate to the low levels of knowledge and practice among mothers in Nigeria compared with the current study where the knowledge levels of mothers were higher. Our study and two other previous studies carried out in Tabouk and another in Mecca seemed to have comparable results regarding common types of food that were given before 6 months of age such as, baby cereals, vegetables and fruits (Alzaheb, 2016; Azzeh, 2017).

In terms of the limitations of this study, it is possible that data collection through online questionnaire could lead some participants to misunderstand some of the questions. However, the questionnaire was tested and readjusted to ensure ease of understanding before it was used. As convenient sampling technique was used, results of this study should not be generalised.

\section{CONCLUSION}

By initiating complementary feeding to their infants before 6 months of age, more than one-third of Saudi mothers who participated in the study were shown to have not followed the relevant WHO recommendation on this matter. As clearly demonstrated, a significant association existed between various KAP aspects in the introduction of early complementary feeding. The knowledge of mothers who practised early complementary feeding had an impact on their attitude and practice as well. Therefore, health care providers should focus on educating mothers to correct their understanding of early complementary feeding and its consequences. It is recommended that further research be undertaken in the different regions of Saudi Arabia in order to obtain better knowledge of the current status of infant feeding among Saudi mothers.

\section{Acknowledgement}

Authors expressed their appreciation to the mothers who participated in the study. This research was funded by the Deanship of Scientific Research at Princess Nourah bint Abdulrahman University through the Fast-track Research Funding Programme.

\section{Authors' contributions}

AA, DA, GA., SA and YA, drafted the article; NB critically revised the article. All authors made a substantial contribution to the design of the work and in the acquisition, analysis and interpretation of data. All authors approved the version that was submitted for publication and participated sufficiently in the work to take public responsibility for appropriate portions of the content.

\section{Conflict of Interests}

The authors declared that there is no conflict of interest. 


\section{References}

Adam EA \& Osama AS (2019). Infant and young child complementary feeding in Saudi Arabia: timely introduction, frequency and diversity. Pak J Nutr 18:216-225.

Al-Awadi FA \& Amine EK (1997). Recent trends in infant feeding patterns and weaning practices in Kuwait. East Mediterr Hlth J 3(3):501-510.

Al-Azzawi SI, Hussein KA \& Shaker NZ (2012). Knowledge, attitude and practices (KAP) of mothers toward infant and young child feeding in primary health care (PHC) centers, Erbil city. Kufa J Nurs Sci 2(2):118-126.

Al-Jassir MS, El-Bashir BM, Moizuddin SK \& AbuNayan AAR (2006). Infant feeding in Saudi Arabia: mothers' attitudes and practices. East Mediterr Hlth J 12(1-2):6-13.

Al-Sairafi M \& Al-Dallal Z (2002). Breastfeeding patterns and practice in the Kingdom of Bahrain (children aged 0-24 months). Kingdom of Bahrain. Ministry of Health. From http:// www.moh.gov.bh. [Retrieved December 6 2018].

Alzaheb RA (2016). Factors associated with the early introduction of complementary feeding in Saudi Arabia. Int $J$ Environ Res Public Hlth 13(7):702.

Azzeh F (2017). Determinants of exclusive breastfeeding and patterns of complementary feeding practices in Mecca city, Saudi Arabia. Int $J$ Child Health Nutr 6(2):80-89.

Batal M, Boulghourjian C \& Akik C (2011). Complementary feeding patterns in a developing country: a cross-sectional study across Lebanon. East Mediterr Hlth J 16(2): 180186.

Berisha M, Ramadani N, Hoxha R, Gashi S, Zhjeqi V, Zajmi D \& Begolli I (2017). Knowledge, attitudes and practices of mothers in Kosova about complementary feeding for infant and children 6-24 months. Med Arch 71(1):37.

Central Statistical Organization (1994). Yemen Demographic and Maternal and Child Health Survey 1991/1992. Sana Yemen. From https: / / dhsprogram.com/pubs / pdf/FR52/ FR52.pdf . [Retrieved December 6 2018].

El Mougi M, Moustafe S, Osman NH \& Ahmed KA (1981). Social and medical factors affecting the duration of breast feeding in Egypt. J Trop Pediatr 27:5-11.
El Mouzan MI, Al Omar AA, Al Salloum AA, Al Herbish AS \& Qurachi MM (2009). Trends in infant nutrition in Saudi Arabia: compliance with WHO recommendations. Ann Saudi Med 29(1):20-23.

Ip S, Chung M, Raman G, Trikalinos TA \& Lau J (2009). A summary of the agency for healthcare research and quality's evidence report on breastfeeding in developed countries. Breastfeed Med 4(S1):S-17-S-30.

Jain S, Thapar RK \& Gupta RK (2018). Complete coverage and covering completely: breast feeding and complementary feeding: knowledge, attitude, and practices of mothers. Med J Armed Forces India 74(1):28-32.

Khattab MS (2000). Cross-sectional study of a child health care programme at one family practice centre in Saudi Arabia. East Mediterr Health $J$ 6(2-3):246-259.

Kittisakmontri K, Fewtrell M, Roekworachai K, Phanpong C \& Lanigan J (2018). Complementary feeding: Attitudes, knowledge and practices of urban families in northern Thailand. Nutr Diet 76(1):57-66.

Launiala A (2009). How much can a KAP survey tell us about people's knowledge, attitudes and practices? Some observations from medical anthropology research on malaria in pregnancy in Malawi. Anthropol Matters J 11(1):1-13.

Manderson L \& Aaby P (1992). An epidemic in the field? Rapid assessment procedures and health research. Soc Sci Med 35(7):839-850.

Olatona FA, Adenihun JO, Aderibigbe SA \& Adeniyi OF (2017). Complementary feeding knowledge, practices, and dietary diversity among mothers of under-five children in an urban community in Lagos State, Nigeria. Inter $J M C H$ AIDS 6(1):46-59.

Pearce J, Taylor MA \& Langley-Evans SC (2013). Timing of the introduction of complementary feeding and risk of childhood obesity: a systematic review. Inter J Obes 37(10):1295.

Radwan H (2013). Patterns and determinants of breastfeeding and complementary feeding practices of Emirati mothers in the United Arab Emirates. BMC Publ Hlth 13(1):1. doi:10.1186/1471-2458-13-171. 
Rao S, Swathi PM, Unnkrishanan B \& Hegade A (2011). Study of complementary feeding practice on mothers of child aged two to two years from coastal south India. Australian Med $J$ 4:252-257.

Reda EB, Teferra AS \& Gebregziabher MG (2019). Time to initiate complementary feeding and associated factors among mothers with children aged 6-24 months in Tahtay Maichew district, northern Ethiopia. BMC Res Notes 12(1): 17 .

Scott JA, Dashti M, Al-Sughayer M \& Edwards CA (2015). Timing and determinants of the introduction of complementary foods in Kuwait: results of a prospective cohort study. $J$ Human Lactation 31(3):467-473.
Shembesh NM, Balo NN \& Singh R (1997). Breastfeeding and weaning patterns in Benghazi Libyan Arab Jamahiriya. East Mediterr Hlth $J$ 3(2):251-257.

Tang L, Lee AH \& Binns CW (2015). Predictors of early introduction of complementary feeding: longitudinal study. Pediatr Inter 57(1):126-130.

WHO (2003). Guiding principles for complementary feeding of the breastfed child. World Health Organization. From https://www.who.int/ nutrition/publications / guiding_principles_ compfeeding_breastfed.pdf. [Retrieved December 4 2018] 